\title{
HABILITACIÓN Y CERTIFICACIÓN PARA LOS PROFESORES EN CHILE
}

Una revisión de las políticas educacionales impulsadas por diversos países nos muestra que quien financia la educación pública ha ido asumiendo un rol crecientemente más protagónico en el diseño de políticas de certificación docente. Éstas buscan asegurar que cada alumno y alumna, cuya educación es subvencionada por el Estado, cuente con profesores que demuestren poseer las competencias mínimas necesarias para desempeñarse bien en la escuela y en el aula. En Chile, los problemas asociados a la calidad de la formación inicial, recientemente tratados en el informe preliminar de la Comisión sobre Formación Inicial Docente (octubre, 2005) y anteriormente identificados en el informe que emitió la Organización para la Cooperación y el Desarrollo Económicos (OCDE) (2004a) sobre la educación chilena, nos llevan a proponer que el Estado también asuma mayor responsabilidad en asegurar la calidad de los nuevos profesores y profesoras que se integren al sistema escolar. Convocando a universidades, sostenedores y profesores se sugiere diseñar e implementar una política de habilitación y acreditación en conjunto, que integre la evaluación docente y el desarrollo profesional.

El desarrollo de políticas para mejorar la calidad de los profesores y de sus programas de formación se sustenta en investigaciones que identifican características docentes — como el estatus de certificación y grado académico en la disciplina que enseñan- correlacionadas positivamente con los resultados de aprendizaje (Darling-Hammond, 2000; Monk, 1994). Estos resultados han sido corroborados por otras investigaciones, por ejemplo, las de Rivkin y Hanushek (2002, citadas en Hannaway \& Woodroffe, 2003), señalando que los progresos observados en el rendimiento en pruebas de matemáticas están asociados a la preparación de los docentes. De acuerdo con otros estudios citados en el mismo artículo, este impacto es aún 
más pronunciado en alumnos con bajo logro académico. Estudios nacionales sobre características de las escuelas efectivas que sirven a alumnos en sectores de pobreza, muestran, entre otros aspectos, que las claves de los buenos resultados tienen sus raíces en la gestión pedagógica y en las prácticas didácticas en el aula (Bellei, Muñoz, Pérez y Raczynski, 2004).

Recientemente, los juicios de calidad de la formación de nuevos profesores en nuestro país están vinculados principalmente al sistema de acreditación. Los sistemas de certificación tienen como objeto de evaluación a las personas y los de acreditación a los programas que ofrecen formación profesional a dichas personas. En nuestra perspectiva, ambos son complementarios, puesto que un programa acreditado nos permite, en principio, inferir que forma egresados de calidad. Sin embargo, considerando los años de acreditación otorgados (de dos a siete años), así como las discrepancias en los factores que consideran los pares evaluadores al emitir su juicio (Téllez, 2005), pensamos que la acreditación, por sí sola, no es suficiente para establecer si los egresados de las carreras de pedagogía están adecuadamente preparados para apoyar el aprendizaje y desarrollo en todos sus alumnos y alumnas, y contribuir al desarrollo de la organización escolar.

\section{La experiencia internacional}

Hasta ahora, la habilitación permanente para trabajar como profesor en Chile se otorga en virtud del título profesional que se obtiene al completar satisfactoriamente todos los requisitos universitarios para las carreras de pedagogía. A partir del trabajo de Larsen, Lock y Lee (2005) podemos identificar otros cinco posibles modelos.

Modelo 1: Habilitación y certificación conjunta con la titulación de una carrera de pedagogía acreditada. El contenido y duración del currículo de formación se ha definido de acuerdo con criterios establecidos por el Estado. 
Modelo 2: Habilitación y certificación conjunta con la titulación de una carrera acreditada, más la rendición y aprobación de exámenes nacionales o estatales. Francia, Alemania, Grecia, Italia, Reino Unido, Japón, Corea del Sur, España, Estados Unidos y México son algunos de los muchos países que exigen a sus nuevos profesores la aprobación de exámenes para obtener la habilitación que los faculta para enseñar en los sistemas públicos (OCDE, 2004b). Esta clase de certificación ha tenido el mérito de explicitar y transparentar las expectativas que la sociedad tiene respecto a competencias mínimas para el ejercicio profesional y ha logrado evaluar el dominio de algunos conocimientos básicos, especialmente de tipo disciplinario. Además, ha respondido a las inquietudes de ciudadanos, padres y apoderados de contar con filtros para asegurar buenos profesores para todos los alumnos.

Sin embargo, también hay evidencia de que la aplicación de exámenes de "lápiz y papel" conlleva problemas y limitaciones, por lo que muchos investigadores cuestionan la pertinencia de utilizar este instrumento como medida única para certificar profesores (Darling-Hammond, 2000; Larsen, Lock y Lee, 2005; Mitchell, Robinson, Plake y Knowles, 2001). Se ha encontrado abundancia de preguntas que sólo miden la memorización de contenidos. El uso de preguntas de selección múltiple es problemático ya que éstas, en el ámbito de la enseñanza, no tienen generalmente una sola respuesta correcta, sino que dependen de los objetivos del currículo, de los aprendizajes que se esperan, de la experiencia previa y de las necesidades de los estudiantes. Por otra parte, no hay consenso respecto de qué debe medirse en estas pruebas. La evidencia respecto del tipo y profundidad del conocimiento que debe demostrar un profesor para lograr aprendizajes significativos en sus alumnos no es conclusiva (Hannaway y Woodroffe, 2003). Para responder a estas limitaciones se han introducido nuevas estrategias evaluativas que permiten retratar el desempeño del docente en contextos y tareas auténticas como, por ejemplo, la elaboración de portafolios y la observación en aula, entre otros. 
Modelo 3: Habilitación y certificación diferida. La habilitación provisional se obtiene al recibir el título profesional, mientras que la certificación permanente sólo después de un número de años de ejercicio profesional, durante el cual se evidencia participación en formación continua. En este proceso, el profesor también entrega pruebas de su desempeño, por las cuales es evaluado.

Modelo 4: Habilitación provisional con la titulación y obtención de la certificación permanente a través de un proceso de evaluación docente durante el primer año de ejercicio. Por ejemplo, Larsen, Lock y Lee (2005) señalan que en Queensland (Australia), después de la habilitación provisional, el profesor ingresa a una escuela por un periodo probatorio de ocho meses. Durante ese tiempo su supervisor, el director del establecimiento, realiza evaluaciones informales y formales de su desempeño en el aula, y, al finalizar el periodo, elabora un informe recomendando continuar o terminar el contrato de trabajo.

Modelo 5: Habilitación provisional e inducción que culmina con evaluación del desempeño docente para los profesores en servicio. En algunos países, la habilitación provisional se basa en completar una carrera de pedagogía, junto con la rendición de exámenes estatales. Este modelo tiene variadas expresiones y se basa en evidencia que señala que los dos primeros años de ejercicio profesional son tremendamente desafiantes para un novato, requiriéndose de un periodo de apoyo estructurado durante su transición desde un rol como "estudiante de la enseñanza" hacia uno de "enseñanza a los estudiantes" (Moskowitz y Stephens, 1997).

En el siguiente apartado proponemos recomendaciones para orientar el diseño de un sistema de la habilitación y certificación de profesores en Chile, que recoge la experiencia internacional que ya hemos resumido. 


\section{Orientaciones de una propuesta para Chile}

En los últimos años se han desarrollado avances importantes en el país, a partir de los cuales se puede construir una política de habilitación y certificación de profesores novatos. Por lo tanto, esta política se concibe como parte integral de un sistema que vincule la acreditación de las carreras de pedagogía con la evaluación del desempeño de los docentes en servicio. Dicha relación debe estar mediada por un compromiso y responsabilidad compartida entre el Ministerio de Educación, las universidades, los sostenedores y los profesores. La experiencia internacional sugiere que la certificación necesita tener un fuerte componente formativo junto con mecanismos de evaluación que otorguen credibilidad y transparencia a los usuarios, en especial a los docentes que buscan la certificación. Asimismo, estos mecanismos deben ser viables, en función de los recursos financieros y el tiempo que se requiere para implementarlo.

Nuestra propuesta reconoce que aprender a enseñar es una tarea compleja, que supone un desarrollo profesional continuo en el cual identificaremos tres etapas de habilitación y certificación: provisional, permanente ${ }^{1}$ y de excelencia. La tabla 1 presenta las etapas que proponemos considerar e identifica dónde se recomienda insertar nuevos mecanismos para fortalecer la evaluación y el apoyo a la inserción de los profesores novatos.

1 La certificación del profesor novato lo habilita a permanecer en el sistema público, a no ser que pierda posteriormente su derecho a ejercer en él por una evaluación insatisfactoria de su desempeño, de acuerdo con la legislación vigente. 
Tabla 1: Etapas del proceso de habilitación y certificación de nuevos profesores

\begin{tabular}{|c|c|c|c|}
\hline Etapa & Mecanismo & Lo existente & Las propuestas \\
\hline 1. Provisional & $\begin{array}{l}\text { - Titulación } \\
\text { de carrera de } \\
\text { pedagogía } \\
\text { acreditada. } \\
\text { - Examen de } \\
\text { conocimientos } \\
\text { disciplinarios y } \\
\text { su didáctica. } \\
\text { - Título y } \\
\text { examen son } \\
\text { conducentes } \\
\text { a habilitación } \\
\text { provisional. }\end{array}$ & $\begin{array}{l}\text { - Acreditación voluntaria. } \\
\text { - Acreditación con } \\
\text { énfasis en procesos. } \\
\text { - Exámenes AEP. } \\
\text { - Propuesta para } \\
\text { elaborar una prueba de } \\
\text { conocimiento } \\
\text { matemático y su } \\
\text { didáctica, (FONDEF). } \\
\text { - Propuesta para } \\
\text { elaborar una prueba } \\
\text { de conocimiento } \\
\text { disciplinario para } \\
\text { profesores de } \\
\text { segundo ciclo básico } \\
\text { (MECESUP). }\end{array}$ & $\begin{array}{l}\text { - Acreditación obligatoria. } \\
\text { - Acreditación que } \\
\text { incorpora criterios } \\
\text { como plan de monitoreo } \\
\text { del logro de las } \\
\text { competencias definidas } \\
\text { en los Estándares de } \\
\text { Desempeño para la } \\
\text { Formación Inicial de } \\
\text { Docentes. } \\
\text { - Crear versión del examen } \\
\text { actualmente en uso } \\
\text { para la Asignación de } \\
\text { Excelencia Pedagógica } \\
\text { (AEP) calibrada para un } \\
\text { profesor novato. }\end{array}$ \\
\hline 2. Permanente & $\begin{array}{l}\text { - Inducción } \\
\text { por dos años, } \\
\text { más resultado } \\
\text { de nivel } \\
\text { "competente" } \\
\text { en la } \\
\text { evaluación del } \\
\text { desempeño } \\
\text { docente } \\
\text { conducentes } \\
\text { a certificación } \\
\text { permanente. }\end{array}$ & $\begin{array}{l}\text { - Evaluación del } \\
\text { desempeño docente } \\
\text { para profesores en } \\
\text { servicio. }\end{array}$ & $\begin{array}{l}\text { - Programa de inducción } \\
\text { diseñado e implementado } \\
\text { en conjunto por } \\
\text { universidades y } \\
\text { sostenedores, por periodo } \\
\text { de dos años con apoyo } \\
\text { de mentores y evaluación } \\
\text { formativa que culmina } \\
\text { con primera evaluación } \\
\text { del desempeño docente. }\end{array}$ \\
\hline 3. Excelencia & $\begin{array}{l}\text { - AEP según } \\
\text { procedimiento } \\
\text { establecido por } \\
\text { el Mineduc. }\end{array}$ & - AEP. & $\begin{array}{l}\text { - Reconocer el sistema } \\
\text { de evaluación de } \\
\text { desempeño AEP } \\
\text { como certificación de } \\
\text { excelencia. } \\
\text { - Incorporar profesores } \\
\text { con AEP y con } \\
\text { desempeño destacado en } \\
\text { la evaluación docente, } \\
\text { como mentores para } \\
\text { apoyar programa de } \\
\text { inducción. }\end{array}$ \\
\hline
\end{tabular}

A continuación, elaboraremos con mayor detalle dos de los aspectos presentados en la tabla anterior: ajustes en el proceso de acreditación y el diseño de programas de inducción para profesores novatos. 


\section{Ajustes en los criterios de acreditación de las carreras de pedagogía}

La primera etapa del sistema que proponemos ya tiene una institucionalidad y trayectoria. De los 342 programas de pedagogía de educación superior, 72 carreras, impartidas por 12 universidades, han obtenido acreditación por periodos que van desde dos a seis años.

La elaboración de los Estándares de Desempeño, desarrollada en el contexto del Programa de Formación Inicial de Docentes, avanzó en la instalación de un sistema de aseguramiento de la calidad de los profesores que ingresan al sistema. Dichos estándares dan respuestas a interrogantes del tipo: ¿cuál es el cuerpo de conocimientos y habilidades que debe sustentar a la profesión docente? ¿Qué debe saber y saber hacer un profesor? Para seguir avanzando, sin embargo, necesitamos formular nuevas preguntas, esta vez respecto de los resultados de los procesos de formación inicial: ¿qué y cuánto saben los egresados acerca de las áreas disciplinarias en las cuales se han preparado para enseñar? ¿Qué logros obtienen los egresados al momento de implementar el currículo escolar con un grupo de alumnos? Un sistema de habilitación y certificación busca responder a este tipo de preguntas.

$\mathrm{Al}$ analizar los nueve criterios que usa la Comisión Nacional de Acreditación (CNAP) para las carreras de pedagogía, concluimos que la mirada del acreditador se orienta hacia la correspondencia entre los procesos y los propósitos que la unidad acreditada ha definido para sí. Usar la acreditación como parte del sistema de habilitación y certificación, sin embargo, supone reorientar esta mirada para destacar los resultados del proceso de formación. Para ilustrar este punto, comparemos muy sucintamente cómo abordan el tema de los resultados los criterios nacionales, por una parte, y los estándares del Consejo Nacional para la Acreditación de la Formación de Profesores en Estados Unidos (National Council for Accreditation of Teacher Education, NCATE), por otra. 
La CNAP lo hace a través del criterio 7 afirmando que: "La unidad debe hacer un seguimiento de sus procesos académicos (tasas de retención, de aprobación, de titulación, tiempo de egreso, niveles de exigencia) e introducir cambios cuando sea necesario". Esta definición claramente deja fuera de la mirada del acreditador el avance de los futuros profesores en cuanto al logro de las competencias profesionales definidas en el perfil de la carrera. Esto llama la atención considerando que el criterio 4 (Perfil Profesional y Estructura Curricular) señala que, al momento de elaborar su perfil profesional y estructurar el currículo, la carrera debe considerar como un elemento "El perfil del/a educador/a egresado/a de la Formación Inicial Docente" (citado en Téllez, 2005).

La evaluación de los futuros profesores aparece en el criterio 6, referido a la Efectividad del Procesos de Enseñanza-Aprendizaje, y requiere de las unidades demostrar la existencia de mecanismos para comprobar el logro de objetivos planteados en el programa de estudios. Aunque no queda explícito, esto parece referirse a los objetivos de las asignaturas de la malla curricular y no a lo definido en el perfil profesional. Sí se pide que se señale, brevemente, cómo la unidad garantiza que sus estudiantes logren una sólida formación de acuerdo con la definición del perfil de egreso. La evaluación del desempeño de los egresados se propone como una actividad de seguimiento una vez que éstos han ingresado al mundo laboral, y no como un criterio para determinar si están preparados para la práctica profesional independiente y, por tanto, en condiciones de recibir su título profesional.

Por otro lado, los criterios de la NCATE se organizan en torno a dos grandes ejes: Capacidad de la Unidad y Desempeño de los Candidatos. El primero se asemeja a los criterios de la CNAP y el segundo difiere sustantivamente. Respecto del segundo criterio se definen dos estándares: uno relacionado con los conocimientos, habilidades y disposiciones de los estudiantes de pedagogía, y el otro con la implementación de un sistema de evaluación para recoger y analizar información acerca de los estudiantes y la unidad, 
clarificando cómo se supervisa el progreso respecto del desempeño de los futuros profesores con relación a los estándares definidos por la profesión, el Estado y la institución.

Para demostrar el cumplimiento de estos dos estándares, el sistema de evaluación debe entregar información que permita examinar: a) la alineación del currículo con los estándares, b) la eficacia de los cursos, las prácticas y los programas, y c) el logro de los conocimientos disciplinarios y si, a su egreso, los candidatos son capaces de implementar una enseñanza que propicie el aprendizaje de sus alumnos. Los criterios que hasta ahora utiliza la CNAP abordan parcialmente los propósitos a) y b). El sistema de certificación que proponemos, sin embargo, requeriría que se incorporen criterios de acreditación para abordar el punto c). Pensamos que una propuesta para la evaluación de los resultados de la formación necesita conciliar la autonomía de cada institución para definir su propio currículo con la existencia de un marco nacional para el sistema escolar, el que todos los profesores deben estar suficientemente preparados para enseñar de acuerdo con los estándares de calidad al que nuestro país aspira.

En cuanto al logro de conocimientos disciplinarios, proponemos la aplicación de un examen nacional, diseñado en conjunto con las universidades, que se aplicaría al finalizar los requisitos de la carrera y previo a la titulación. Sus resultados serían parte de la información que la unidad entregaría con relación a los resultados de sus procesos de formación, junto con las opiniones de los estudiantes y empleadores que se solicitan actualmente.

Respecto de la capacidad de los egresados para implementar una enseñanza que propicie el aprendizaje y desarrollo de sus alumnos, las unidades deben recoger y entregar evidencia válida y confiable acerca del desempeño de los futuros profesores en contextos escolares. Esta información podría obtenerse a través de la implementación de un sistema de evaluación riguroso y sistemático del eje curricular de práctica progresiva, en donde el estudiante de 
pedagogía va asumiendo crecientes niveles de responsabilidad al enseñar a un grupo curso. Sin embargo, como lo consigna el informe de la OCDE (2004a), las carreras de pedagogía actualmente carecen de un monitoreo riguroso de las experiencias de práctica.

En nuestro país, investigadores de tres universidades están abordando esta falencia a través del diseño y validación de una metodología que permita evaluar el desempeño de los alumnos en su práctica profesional, enfocada en su impacto en los aprendizajes del grupo curso ${ }^{2}$. Para ello se ha desarrollado un instrumento de Muestras de Desempeño Docente (MDD) que define seis estándares y representa una adaptación del modelo Teacher Work Sample Methodology propuesto por Shalock (1998). Éste entrega a los practicantes una metodología para planificar una unidad de enseñanza-aprendizaje que dure de seis a diez sesiones de clases. Junto con entregar orientaciones específicas para desarrollar tareas definidas y evidenciar el logro de cada estándar, entrega una rúbrica que describe tres niveles de calidad del desempeño en esas tareas. La MDD pide al futuro profesor comunicar su razonamiento pedagógico y reflexión sobre sus decisiones al momento de planificar, implementar y evaluar el impacto de su enseñanza, lo que es consistente con lo que le solicitará el proceso de la evaluación del desempeño docente cuando esté ejerciendo su profesión. Al contar con un instrumento válido y confiable, un análisis de los resultados agregados para una cohorte entrega otro indicador del impacto de los procesos de formación.

2 Proyecto FONDECYT 1040020 "Validación de la Metodología: Muestras del Trabajo Docente para la Evaluación por Estándares de Desempeño en la Formación Inicial de Profesores". Desarrollado por investigadores de la Pontificia Universidad Católica de Valparaíso, Pontificia Universidad Católica de Chile y Universidad de Atacama. 


\section{Inducción de profesores novatos}

Hasta el momento hemos abordado el aspecto de evaluación asociado a la certificación, pero reconocemos que la evaluación, por sí sola, no conduce a un mejoramiento de la formación docente. Las investigaciones demuestran que, no obstante los esfuerzos por lograr una buena calidad de la formación inicial, el desempeño cotidiano en contextos reales presenta al profesor principiante muchos desafíos, no anticipados, que requieren de reflexión con profesionales insertos en el sistema educativo y en el aula. Para ello se propone la incorporación de un proceso de inducción que ofrezca un apoyo estructurado a los profesores que ingresan al mundo laboral. De esta manera, se le ofrecería, desde el inicio, un sistema de apoyo para continuar desarrollando las competencias mínimas que el contexto de la escuela requiere para desempeñarse bien.

El estudio de Moskowitz y Stephens (1997), realizado en 11 países de la $\mathrm{APEC}^{3}$, nos permite identificar cuatro características de programas de inducción efectivos, convenientes de tomar en cuenta al diseñar un sistema para Chile. Estas son:

a) Las autoridades proveen de suficiente apoyo político y financiero y las escuelas de tiempo, entre otros recursos. Esto implica el diseño de una política de desarrollo profesional docente que contemple recursos para la inducción de nuevos profesores, los que podrían asignarse mediante un fondo concursable al cual podrían postular consorcios formados por universidades y sostenedores. Contar con universidades y colegios que asuman el desarrollo profesional del novato como una responsabilidad compartida, tendría impacto en mejorar la calidad de los establecimientos educacionales y en retroalimentar los procesos de formación.

b) Las metas de la inducción están claramente articuladas con las necesidades del sistema escolar y con las de cada profesor novato. Éstas no sólo se refieren al propósito de certificar si los

3 Asia-Pacific Economic Cooperation. 
profesores novatos pueden cumplir con las tareas docentes, sino también al de promover el desarrollo de los profesionales y de la organización escolar. Las experiencias exitosas muestran que la inducción cumple con los siguientes objetivos: socializar al novato en las responsabilidades, procedimientos y cultura de la institución escolar, apoyando su transición al mundo laboral, y fortalecer sus competencias profesionales y sentido de eficacia, para proteger al nuevo profesor contra la deserción temprana de la profesión docente.

c) El apoyo al profesor novato contempla una combinación de estrategias que incentivan su interacción con profesores experimentados: mentores, modelos de buenas prácticas, talleres y evaluación formativa. Enfatizar la evaluación formativa por sobre la sumativa, durante el periodo de inducción, incentiva al nuevo profesor a tomar riesgos y a pedir ayuda, aspectos que podrían inhibirse si se pone demasiado énfasis en la evaluación sumativa con consecuencias para su empleabilidad. Después de un tiempo de aprendizaje profesional, que puede extenderse hasta por dos años, recomendamos que se evalúe su desempeño por agentes externos a la escuela, de acuerdo con el Sistema Nacional de Evaluación de Desempeño Docente. La obtención de un resultado de desempeño de nivel "competente" o "superior" le daría derecho a obtener la certificación permanente por parte del Estado.

d) Es necesario distinguir las responsabilidades del profesor novato de las que deben esperarse de profesores con certificación permanente y de excelencia. En algunos países, por ejemplo, se asignan al profesor novato cursos que se estiman menos desafiantes y se reduce su carga horaria para que pueda involucrarse en actividades de desarrollo profesional asociadas a la inducción. Por otra parte, al profesor con certificación de excelencia (AEP) o con desempeño de nivel "destacado" se le da la oportunidad de cumplir tareas como profesor mentor a cargo del apoyo a profesores novatos durante el periodo de inducción, etapa que involucra múltiples oportunidades de reflexión contribuyendo al crecimiento profesional de ambos. 


\section{Conclusiones}

Este esquema recoge las necesidades detectadas en diversos estudios nacionales, que nos alertan sobre la necesidad de avanzar en el aseguramiento de la calidad de los profesores que ingresan a trabajar al sistema escolar subvencionado con fondos públicos. Ello supone, por una parte, fortalecer el sistema de evaluación y, por otra, mejorar la relación entre la formación inicial y el desarrollo profesional continuo de los docentes. Entendemos que la propuesta que eventualmente se llegue a implementar surgirá de un consenso entre actores clave del sistema escolar: Ministerio de Educación, Colegio de Profesores, universidades y representantes de sostenedores.

Hemos propuesto un sistema de habilitación y certificación para un profesor novato que considera tres etapas, basadas en tres componentes interrelacionados y construidas a partir de instrumentos existentes: (1) ajustes a los criterios de acreditación de carreras de pedagogía, fortaleciendo la mirada sobre la evaluación de los aprendizajes y desempeño de sus egresados, (2) diseño de programas de inducción para el profesor novato que pueden ser implementados conjuntamente entre universidades y sostenedores, que culmina en (3) el logro de un nivel competente en la evaluación docente al finalizar el periodo de inducción.

La experiencia internacional nos señala la importancia de instalar desde el diseño de la política el monitoreo, para determinar hasta qué punto cumple con los propósitos de asegurar que todos los niños y jóvenes de nuestro país tengan acceso a buenos profesores. Además, nos indica que se necesita velar por que llegue a todos los profesores y no sólo a quienes trabajan en centros urbanos cercanos a las universidades. El desafío es generar un sistema que incentive el aprendizaje profesional y que vaya más allá de ser un instrumento público por medio del cual el Estado certifica calidad. 


\section{Referencias bibliográficas}

Bellei, C., Muñoz, G., Pérez, L. M., Raczynski, D. ¿Quién dijo que no se puede? Escuelas efectivas en sectores de pobreza. UNICEF-Gobierno de Chile, 2004. Disponible en http://www.asesoriasparaeldesarrollo. $\mathrm{cl} /$ secciones/documentos/escuelas_efectivas_sectores_pobreza.doc

Comisión Nacional de Acreditación (CNAP). Guía de Formularios para la Acreditación de Carreras. Disponible en http://www.cnap.cl/Acr_ Carreras/criterios/critesp/crit_esp_f.html

Comisión sobre Formación Inicial Docente (6 y 7 de octubre, 2005). Informe preliminar de la Comisión sobre Formación Inicial Docente. Documento de Trabajo Encuentro Nacional: Propuestas de Políticas para la Formación Docente. Universidad Metropolitana de Ciencias de la Educación, Santiago de Chile, 2005.

Darling-Hammond, L. Teacher quality and student achievement: A review of state policy evidence. Education Policy Analysis Archives, 8(1), 2000, pp. 1-49.

Hannaway, J., \& Woodroffe, N. Policy Instruments in Education. In: R. Floden (ed.), Review of Research in Education (Vol. 27, pp. 1-24). American Educational Research Association, Washington DC, 2003.

Larsen, M., Lock, C., \& Lee, M. Professional Certification and Entry-ToPractice Assessments. A Report for the Teaching Policy and Standards Branch Ontario Ministry of Education, 2005. Available at http:// publish.edu.uwo.ca/marianne.larsen/larsen.pdf

Mitchell, K. J., Robinson, D. Z., Plake, B. S. \& Knowles, K. T., (eds). Testing Teacher candidates: The role of licensure tests in improving teacher quality. National Academy of Science; Washington DC. 2001. Available at http://books.nap.edu/catalog/10090.html

Monk, D. H. Subject matter preparation of secondary mathematics and science teachers and student achievement. Economics of Education Review, 13 (2), 1994, pp. 125-145.

Moskowitz, J. \& Stephens, M., (eds). From Students of Teaching to Teachers of Students:Teacher Induction Around the Pacific Rim. For Asia Pacific Economic Cooperation APEC Education Forum, U.S. Department of Education, 1997. Available at http://www.ed.gov/ pubs/APEC/preface.html 
National Council for Accreditation of Teacher Education (NCATE). Professional Standards Accreditation of Schools, Colleges, and Departments of Education. The Standard of Excellence in Teacher Preparation, 2002 Edition. Washington, DC. Available at http://www. ncate.org/documents/unit_stnds_2002.pdf

OCDE Revisión de políticas públicas: Chile. Organization for Economic Cooperation and Development: Paris, 2004a.

OCDE Teachers Matter: Attracting, Developing, and Retaining Effective Teachers. Synthesis Report. Organization for Economic Cooperation and Development: Paris, 2004b.

Rivkin, S. G. \& Hanushek, E. A. Teacher quality in Texas: Using school administrators' data to understand alternative policy proposals. Paper presented at the Instructional performance consequences in highpoverty schooling: Research Seminar II, Washington DC, 2002.

Schalock, M.D. Accountability, student learning, and the preparation and licensure of teachers: Oregon's teacher work sample methodology. Journal of Personnel Evaluation in Education, 12(3), 1998, pp. 269286.

Téllez, F. Caracterización de la formación inicial de las carreras de pedagogía de las universidades chilenas que han participado en el proceso de acreditación de la Comisión Nacional de Acreditación de Pregrado (CNAP). Trabajo presentado en el Encuentro Nacional: Propuestas de Políticas para la Formación Docente. Universidad Metropolitana de Ciencias de la Educación, Santiago de Chile, octubre 2005. 